\title{
High-risk Human Papillomavirus Genotypes in Cervical Lesions and Vaccination Challenges in China
}

\author{
Qiu-Xiang Xu, Zhen-Yu Zhang*
}

\begin{abstract}
Cervical cancer, mostly progressing from cervical intraepithelial neoplasia, is a major cause of morbidity and mortality in Chinese women. This is largely due to high prevalence of high-risk human papillomaviruses (hr-HPVs) in the population. The prevalence of hr-HPV DNA in women and in cervical lesions women ranged from $9.9 \%$ to $17 . \%$ and from $50.5 \%$ to $\mathbf{7 0 . 9 \%}$ in different regions of China, respectively. The most common genotypes somewhat differ between regions throughout the country and from those in many other countries. This may be a challenge to cervical cancer screening and prevention in China. Combined detection of particular HPV genotypes should be recommended in all geographical regions in China and greater attention must be paid to specific hr-HPV types during cervical cancer screening and follow-up of cervical lesions. Besides, vaccination for prevention of cervical cancer by particular HPV genotypes, has not been introduced to China so far. Updated knowledge on prevalent HPV genotypes should be provided to public health organizations to help with the development of more effective HPV vaccines, which can protect Chinese women against HPV types prevalent in local China and thus have a substantial impact on the cervical cancer burden.
\end{abstract}

Keywords: Cervical cancer - cervical intraepithelial neoplasia - China - HPV - HPV genotype - vaccine

Asian Pac J Cancer Prev, 16 (6), 2193-2197

\section{Introduction}

Cervical cancer is the second most prevalent malignancy in women worldwide and there were up to nearly 528,000 new cases and about 266,000 deaths in 2012 (International Agency for Research on Cancer, 2014). In developing countries, including China, it's the second most common cause of cancer-related mortality among women (Jemal et al., 2011). Cervical cancer is mostly progressed from cervical intraepithelial neoplasia (CIN). The morbidity of CIN and cervical cancer, which are referred to as cervical lesions, was about $5.8 \%$ to $6.5 \%$ in China (Li et al., 2010; Zhao et al., 2012).

The central cause of cervical lesions is clearly verified to be human papillomavirus (HPV) infection, especially high-risk HPV (hr-HPV), by epidemiological studies. HPV DNA can be identified in almost all (99.7\%) cases of cervical cancer in studies from 22 countries (Walboomers et al., 1999) and the most prevalent HPV genotypes are HPV16 and 18 worldwide (Clifford et al., 2003; Smith et al., 2007; Sanjose et al., 2010). However, it's likely to be somewhat different in China according to certain newly done studies (Hou et al., 2012; Wang et al., 2013; Zhang et al., 2013; Ding et al., 2014; Jing et al., 2014). Besides, vaccine, preventing cervical cancer from particular HPV genotypes, has not been introduced to China so far.
Hence, it's necessary to get more updated knowledge on the distribution characteristics of hr-HPV genotypes among cervical lesions women in China and this will have profound guiding significance in cervical cancer prevention.

\section{Burden of cervical lesions in China}

China is a developing country located in eastern Asia, with the land area of about 9.6 million square kilometers and the population of as many as one quarter of that of the world. The burden of cervical lesions is disproportionately high in developing countries. In China, the incidence of cervical cancer is high, with about 132,300 new cases each year (Kim et al., 2009). And the prevalence of cervical lesions ranged from $2.5 \%$ to $5.9 \%$, according to some latest cervical cancer screening surveys from different provinces (Zhang et al., 2008; Zhao et al., 2009; Li et al., 2010; Zhang et al., 2013). All these suggest cervical lesions is an important reproductive health problem in China. And in a pooled analysis of 17 population-based studies, the morbidity of cervical lesions was up to about $6.5 \%$ and the age-standardized prevalence was $3.1 \%$ for cervical intraepithelial neoplasia grade 1 (CIN1), $1.3 \%$ for CIN2, $1.2 \%$ for CIN3+ (Zhao et al., 2012). They indirectly proved that the cervical cancer burden in 
China is under-reported and documented the inadequacy of current cervical cancer screening in China. Hence, the exact number of women suffering from cervical lesions is likely to be a little higher than that found by these studies.

\section{Prevalence of hr-HPV in cervical lesions among Chinese women}

Epidemiological studies have clearly verified that the central cause of cervical lesions is HPV infection, especially hr-HPV, which is considered to be oncogenicity. HPV can be identified in almost all $(99.7 \%)$ cases of cervical cancer in studies from 22 countries (Walboomers et al., 1999). Hr-HPV DNA was positive in about $90 \%$ of high-grade cervical lesions in US (Wright et al., 2012). Some Chinese cervical cancer screening studies found that the positive rate of hr-HPV infection among women ranged from $9.9 \%$ to $16.97 \%$ ( $\mathrm{Li}$ et al., 2010; Ye et al., 2010; Chen et al., 2013; Mai et al., 2014) and were even as high as $33.2 \%$ in women with high-risk factors (Zhang et al., 2008). The prevalence of hr-HPV in cervical lesions were reported to be $50.5 \%$ to $70.9 \%$ in cervical lesion cases (Zhang et al., 2008; Li et al., 2010). And the positive rate of hr-HPV in CIN1 and CIN2+ ranged from $40.3 \%$ to $68.8 \%$, from $72.3 \%$ to $88.6 \%$, respectively (Zhang et al., 2008; Li et al., 2010; Liu et al., 2010). As high as $94.1 \%$ of the prevalence in cervical cancer were reported (Zhang et al., 2008). In our recent multicenter study in China, in which 2207 CIN2+ patients were enrolled, hrHPV positive rate in CIN 1 and CIN 2+ were $78.20 \%$ and $92.80 \%$, respectively. However, the manuscript of this study is just being submitted.

As China covers a large area, with 5,200 kilometers from eastern border to western and 5,500 kilometers from south to north, the prevalence of hr-HPV may vary between geographic locations. Keith W.K. LO compared the heterogeneity of HPV prevalence in cervical cancer specimens collected from 5 parts of China and it suggested no significant difference between regions (Lo et al., 2002). However, more than twelve years have passed since their assessment, a new analysis should be carried out to estimate the heterogeneity of hr-HPV prevalence between different locations according to the latest studies. Besides, just as confirmed, HPV prevalence highly depends on the detection method and the prevalence was previously underestimated (Lo et al., 2002; Li et al., 2008). What's more, bias would arise in terms of overestimating the prevalence of hr-HPV infection if local researcher selected patients on the basis of CIN grade (e.g. hr-HPV DNA is more prevalent in CIN3 than in CIN2). With or without significant difference in the prevalence of hr-HPV between regions, these studies all provided high prevalence of hrHPV in cervical lesions.

\section{Most prevalent hr-HPV genotypes in cervical lesions in China}

According to many population-based cervical cancer screening studies from different country of the world, HPV16 was the most prevalent types in cervical lesions (Kjær et al., 2008; Onuki et al., 2009; Sjoeborg et al., 2010;
Hou et al., 2012; Wright et al., 2012). In Copenhagen, Denmark, HPV16, 31, and 33, in order of decreasing prevalence, were the most prevalent types in CIN3 (Kjær et al., 2008). In its nearby country Norway, HPV 16 was also followed by HPV31 and 33 among CIN2+ cases (Sjoeborg et al., 2010). While in Japan, to the east of China, HPV52 and 58, following HPV16, were the most prevalent types in CIN2-3 (Onuki et al., 2009). It seems that the most prevalent hr-HPV types in cervical lesions vary between geographic locations. And there did be variation when ranking of individual hr-HPV types, except HPV16 and 18, across countries (Sanjose et al., 2010; Li $\mathrm{N}$ et al., 2011). We eagerly want to know the detailed prevalent HPV types in China and select studies carried out lately, in which cervical lesions were confirmed by pathology not based on exfoliative cervical cells but samples obtained through biopsy or surgery, gold criteria for diagnosis of cervical lesions. However, there were not many such studies in China.

In a study carried out more than ten years ago, HPV16 and 18 were the most prevalent genotypes in cervical cancer (Lo et al., 2002). In the latest studies, the most prevalent hr-HPV sub-types in cervical lesions, including cervical cancer, varied somewhat between different locations and even between different studies in a same region. In Beijing, a single center study, with 341 cervical lesion cases, reported that HPV16, 58, 66, 18 and 33 were most common hr-HPV in CIN1, and HPV16, $33,58,52$ and 31 were most prevalence in CIN2+ (Hou et al., 2012). Another single-center study, with 1,082 patients, found that the most common hr-HPV genotypes in cervical lesions and cervical cancer were HPV16, 52, 58, 33, 18 and HPV16, 33, 18, 52, 58, respectively (Ding et al., 2014). A multiple-center study in Beijing showed that HPV16, 58, 33, 56, and 31 were the most frequent genotypes in cervical lesions (Li et al., 2010). In another east part of China, a study showed that the most common genotypes were HPV16, 58, 33,52 and 66 in CIN patients (Wang et al., 2013). In southwestern China, HPV16, 58, 18,52 and 33 were the most prevalent genotypes in cervical cancer (Li et al., 2011).

It's noted from these results that HPV18, 31, 33, 52, 58, 56 and 66 were prevalent types, as well as HPV16, among women with cervical lesions in China. And the specific prevalent type, except HPV16 and 58, in different studies or between different provinces varies somehow. Additional, HPV58, 52 types were more prevalent in China than other regions throughout the world. This coincides with the finding of a meta-analysis conducted by Chan PKS who reported that HPV52 and 58 shared a higher prevalence and attribution among CIN in Eastern Asia (Chan et al., 2014). Besides, Zhang R observed some differences in the type distribution of urban verses rural areas, who described like this: the prevalence of HPV58 in urban areas was more than double that in rural areas, and HPV59 was the sixth most prevalent type in urban areas, but not even in the top ten most prevalent types in rural areas (Zhang et al., 2013). In China, rural population accounts for a large proportion and the ratio of rural population to urban differs between provinces. This may be an important reason for the difference when ranking 
the most common HPV types between different provinces. And this indicates the importance of stratified sampling in the study. What's more, as mentioned before, bias would also arise in terms of overestimating the prevalence of particular sub-type if local researcher selected patients on the basis of CIN grade. More accurate result can be obtained only when researchers analyze the positive rate in each detailing disease grade and histologic type should be taken into consideration. (e.g. CIN1, CIN2, CIN3, squamous cervical cancer (SCC) and adenocarcinoma of cervix (ACC)). Lastly, the answer to the question that does the prevalence of particular HPV genotype differ between geographic locations nowadays needs to be confirmed in high-quality analysis or multicenter study throughout the country.

Just as known, the most common HPV types vary between different grade of cervical lesions or histologic type and individual HPV type shared a particular prevalence and attribution among different grade of cervical lesions or histologic type. In cervical cancer, HPV18 is more prevalent than in low grade cervical lesions among Chinese women (Wu et al., 2009; Zhao et al., 2009; Ding et al., 2014), which coincides with the result of a multicenter US cervical cancer screening trial (Wright et al., 2012). And some researchers verified that HPV18 was more prevalent in ACC than in SCC (Clifford et al., 2003; Smith et al., 2007) and implied that HPV 18 genotyping could be useful in identifying adenocarcinoma in situ (Wright et al., 2012). The predominant roles of HPV18, along with HPV16, in invasive carcinomas were confirmed. Besides, HPV 18 has been shown to be a more rapid transition to malignancy (Burger et al., 1996) and a poorer prognosis of cancer patients (Hildesheim et al., 1999; Schwartz et al., 2001), compared to HPV16.

In all, combined detection of particular HPV genotypes should be recommended to corresponding geographical regions in China, according to above studies. But it's convenient to detect all hr-HPV in one same sample at the same time nowadays and it won't cost much more. So genotyping of nearly all hr-HPV types when testing is widely used. And during cervical cancer screening and follow-up of cervical lesions in China, greater attention must be paid to specific hr-HPV genotypes, e.g. HPV58 and 52, compared to many other countries.

\section{Risk factors of cervical lesions in China}

The essential cause of cervical caner is clearly verified to be hr-HPV persistent infection. During the progression from CIN to cervical cancer, there are a series of four steps - HPV transmission, viral persistence, progression of a clone of persistently infected cells to precancer, and invasion. Any factors boost the progression of above steps may be risk factors of cervical lesions. And there are a number of risk factors that predispose women to acquiring hr-HPVs (Schiffman et al., 2007). The factors include marital status, multiple sexual partners, more number of husband's sexual partners, no condom use, early age of first intercourse, early age at first pregnancy (Chou 1991; Zhao et al., 2009; Li et al., 2010). In Changdong Li's research, high education levels, usage of oral steroid contraceptives, smoking, poor genital hygiene, columnar ectopy, infected with other sexually transmitted pathogens such as trichomonas vaginitis or herpes simplex virus were considered to be risk factors (Li et al., 2010). And Jing Ye's findings are inconsistent with theirs in smoking, reproductive history and condom use (Ye et al., 2010). However, a latest large prospective study confirmed the role of tobacco smoking as an important risk factor for both CIN3 and invasive cervical cancer (Roura et al., 2014). Another study provided some evidence that conscientious condom use promoted by health education programs could reduce the risk of cervical cancer at the population level (Shepherd et al., 2011). In addition, there are other co-factors such as immunodeficiency and host genetic factors which are also important for progression of cervical cancer (Moore et al., 2012).

\section{HPV vaccination and challenges in China}

So far, two kinds of prophylactic HPV vaccines has been developed to prevent cervical cancer, including Cervarix ${ }^{\circledR}$ HPV vaccines (preventing mainly HPV16 and 18 ) and quadrivalent Gardasil@ HPV vaccines (active against HPV 6, 11, 16 and 18) (Kawana et al., 2009). Gardasil was originally licensed for prevent against premalignant genital lesions and cervical cancer causally related to certain oncogenic HPV types. Being effective and efficacious in preventing cervical cancer, they are licensed and available in most countries worldwide but the mainland China. It's eagerly hoped that HPV vaccines can be available in China soon. However, there is likely to be several challenges during their introduction to China.

The two vaccines above only target HPV6, 11, 16, and 18 . So it seems that the vaccines won't be effective in preventing against other oncogenic hr-HPV sub-types which are also prevalent in mainland China. According to the studies mentioned above, hr-HPV58, 52, 31, 33, 56 and 66 were also detected in a substantial proportion of cervical lesions women ( $\mathrm{Li}$ et al., 2010; Li et al., 2011; Hou et al., 2012; Wang et al., 2013; Ding et al., 2014). Women infected with such hr-HPV types may not be protected from developing cervical lesions by the current two vaccines. And the same challenge came about in some other countries such as Denmark, Japan, Cameroun and Zambia, where non-16 and 18 subtypes were prevalent (Sahasrabuddhe et al., 2007; Kjær et al., 2008; Desruisseau et al., 2009; Onuki et al., 2009). The challenge therefore presents the urgency of developing HPV vaccines for particular genotypes prevalent in these countries. And there may not be a long way before effective prophylactic vaccines are available in the mainland China. A new nanovalent vaccine, protecting against HPV6, 11, 16, 18, $31,33,45,52$ and 58, is in clinical trials now (Drolet et al., 2014). And it will be more useful in China and other countries where some non-16 and 18 sub-types were prevalent.

What's more, the targeting group for HPV vaccination is needing defined. Girls, before the onset of sexual activities, should be targeted for HPV vaccination, generally (Agosti et al., 2007). And now, universal vaccination (vaccinating both men and women) is 
recommended in several countries, since abundant evidence is emerging of its effectiveness in preventing HPV-related conditions in men. However, the results of cost-effectiveness of universal HPV vaccination have been mixed and much work is needed in the future (Marsh et al., 2014). Another challenge to HPV vaccination in China is the prohibitive cost. In China, a developing country, there were more than 1.3 billion people totally, of which about half are women. If more girls and women are to be reached, the government or donor funding may have to subsidize a large number of vaccines, which will do cost a lot.

Although with some challenges, the prevention and control of cervical lesions, a preventable disease, through screening and HPV vaccination should be a national priority to make sure that more women are under protection against doubtless causal agents.

\section{References}

Agosti JM, Goldie SJ (2007). Introducing HPV vaccine in developing countries-key challenges and issues. $N$ Engl $J$ Med, 356, 1908-10.

Burger RA, Monk BJ, Kurosaki T, et al (1996). Human papillomavirus type 18: association with poor prognosis in early stage cervical cancer. J Natl Cancer Inst, 88, 1361-8.

Chan PKS, Ho WCS, Chan MCW, et al (2014). Meta-analysis on prevalence and attribution of human papillomavirus types 52 and 58 in cervical neoplasia worldwide. PLoS One, 9, 107573.

Chen ZF, Meng W, Du R, et al (2013). Genotype distribution and the relative risk factors for human papillomavirus in Urumqi, China. Exp Ther Med, 6, 85-90.

Chou P (1991). Review on risk factors of cervical cancer. Zhonghua Yi Xue Za Zhi, 48, 81-8.

Clifford GM, Smith JS, Plummer M, Munoz N, Franceschi S (2003). Human papillomavirus types in invasive cervical cancer worldwide: a meta-analysis. Br J Cancer, 88, 63-73.

Desruisseau AJ, Schmidt-Grimminger D, Welty E (2009). Epidemiology of HPV in HIV-positive and HIV-negative fertile women in Cameroon, West Africa. Infect Dis Obstet Gynecol, 2009, 810596.

Ding X, Liu Z, Su J, et al (2014). Human papillomavirus typespecific prevalence in women referred for colposcopic examination in Beijing. J Med Virol, 86, 1937-43.

Drolet M, Laprise JF, Boily MC, et al (2014). Potential costeffectiveness of the nonavalent human papillomavirus (HPV) vaccine. Int J Cancer, 134, 2264-8.

Hildesheim A, Hadjimichael O, Schwartz PE, et al (1999). Risk factors for rapid-onset cervical cancer. Am J Obstet Gynecol, 180, 571-7.

Hou R, Xu CY, Zhang SW, Wu MH, Zhang WY (2012). Distribution of human papillomavirus genotype and cervical neoplasia among women with abnormal cytology in Beijing, China. Int J Gynaecol Obstet, 119, 257-61.

International Agency for Research on Cancer (2014). GLOBOCAN 2012: Estimated cancer incidence, mortality and prevalence worldwide in 2012. Available: http:// globocan.iarc.fr/Pages/fact_sheets_cancer.aspx.

Jemal A, Bray F, Center MM, et al (2011). Global cancer statistics. CA Cancer J Clin, 61, 69-90.

Jing LP, Zhong XM, Huang WH, et al (2014). HPV genotypes and associated cervical cytological abnormalities in women from the pearl river delta region of guangdong province, China: a cross-sectional study. BMC Infect Dis, 14, 388-96.

Kawana K, Yasugi T, Taketani Y (2009). Human papillomavirus vaccines: current issues and future. Indian J Med Res, 130, 341-7.

Kim K, Zang R, Choi SC, et al (2009). Current status of gynecological cancer in China. J Gynecol Oncol, 20, 72-7.

Kjær SK, Breugelmans G, Munk C, et al (2008). Populationbased prevalence, type-and age-specific distribution of HPV in women before introduction of an HPV-vaccination program in Denmark. Int J Cancer, 123, 1864-70.

Li CD, Wu MH, Wang JD, et al (2010). A population-based study on the risks of cervical lesion and human papillomavirus infection among women in beijing, people's republic of China. Cancer Epidemiol Biomarkers Prev, 19, 2655-64.

Li JK, Zhang D, Zhang Y, et al (2011). Prevalence and genotype distribution of human papillomavirus in women with cervical cancer or high-grade precancerous lesions in Chengdu, western China. Int J Gynecol Obstet, 112, 131-4.

Li N, Franceschi S, Howell-Jones R, Snijders PJ, Clifford GM (2011). Human papillomavirus type distribution in 30,848 invasive cervical cancers worldwide: Variation by geographical region, histological type and year of publication. Int J Cancer, 128, 927-35.

Li Y, Wang YP, Jia CW, et al (2008). Detection of human papillomavirus genotypes with liquid bead microarray in cervical lesions of northern Chinese patients. Cancer Genet Cytogenet, 182, $12-7$.

Liu X, Zhang SL, Ruan Q, et al (2010). Prevalence and type distribution of human papillomavirus in women with cervical lesions in liaoning province, China. Int J Gynecol Cancer, 20, 147-53.

Lo KWK, Wong YF, Chan MKM, et al (2002). Prevalence of human papillomavirus in cervical cancer: a multicenter study in China. Int J Cancer, 100, 327-31.

Mai RQ, Huang B, Shen L, et al (2014). Genotype distribution of human papillomavirus in women with abnormal cervical cytology in an esophageal carcinoma high incidence area of China. Asian Pac J Cancer Prev, 15, 4945-50.

Marsh K, Chapman R, Baggaley RF, Largeronc N, Bresse $X$ (2014). Mind the gaps: What's missing from current economic evaluations of universal HPV vaccination? Vaccine, 32, 3732-9.

Moore EE, Wark JD, Hopper JL, et al (2012). Study Group: The roles of genetic and environmental factors on risk of cervical cancer: a review of classical twin studies. Twin Res Hum Genet, 15, 79-86.

Onuki M, Matsumoto K, Satoh T, et al (2009). Human papillomavirus infections among Japanese women: agerelated prevalence and type-specific risk for cervical cancer. Cancer Sci, 100, 1312-6.

Roura E, Castellsague X, Pawlita M, et al (2014). Smoking as a major risk factor for cervical cancer and pre-cancer: results from the EPIC cohort. Int J Cancer, 135, 453-66.

Sahasrabuddhe VV, Mwanahamuntu MH, Vermund SH, et al (2007). Prevalence and distribution of HPV genotypes among HIV-infected women in Zambia. Br J Cancer, 96, 1480-3.

Sanjose SD, Qunit GVW, Alemany L, et al (2010). Human papillomavirus genotype attribution in invasive cervical cancer: a retrospective cross-sectional worldwide study. Lancet Oncol, 11, 1048-56.

Schiffman M, Castle PE, Jeronimo J, et al (2007). Human papillomavirus and cervical cancer. Lancet, 370, 890-907.

Schwartz SM, Daling JR, Shera KA, et al (2001). Human papillomavirus and prognosis of invasive cervical cancer: a population-based study. J Clin Oncol, 19, 1906-15.

Shepherd JP, Frampton GK, Harris P (2014). Interventions for encouraging sexual behaviours intended to prevent cervical cancer. Cochrane Database Syst Rev, 4, 1035. 
Sjoeborg KD, Trope A, Lie AK, et al (2010). HPV genotype distribution according to severity of cervical neoplasia. Gynecol Oncol, 118, 29-34.

Smith JS, Lindsay L, Hoots B, et al (2007). Human papillomavirus type distribution in invasive cervical cancer and high-grade cervical lesions: a meta-analysis update. Int J Cancer, 121, 621-32.

Walboomers JM, Jacobs MV, Manos MM, et al (2012). Human papillomavirus is a necessary cause of invasive cervical cancer worldwide. J Pathol, 189, 12-9.

Wang L, Dai SZ, Chu HJ, Cui HF, Xu XY (2013). Intergration sites and genotype distributions of human papillomavirus in cervical intraepithelial neoplasia. Asian Pac J Cancer Prev, 14, 3837-41.

Wright TC, Stoler MH, Behrens CM, et al (2012). The ATHENA human papillomavirus study: design, methods, and baseline results. Am J Obstet Gynecol, 206, 46.

Wu EQ, Yu XH, Zha X, et al (2009). Distribution of human papillomavirus genotypes in archival cervical lesions in eastern inner mongolian autonomous region, China. Int $J$ Gynecol Cancer, 19, 919-23.

Ye J, Cheng XD, Chen XJ, et al (2010). Prevalence and risk profile of cervical human papillomavirus infection in Zhejiang Province, southeast China: a population-based study. Virol J, 7, 66-76.

Zhang R, Velicer C, Chen W, et al (2013). Human papillomavirus genotype distribution in cervical intraepithelial neoplasia grade 1 or worse among 4215 Chinese women in a population-based study. Cancer Epidemiol, 37, 939-45.

Zhang WY, Xue YZ, Chen M, Han L, Luo M (2008). Prevalence of high-risk human papillomavirus infection in different cervical lesion among organized health-examination women in Shanghai, China. Chin Med J (, 121, 1578-82.

Zhao FH, Lewkowitz AK, Hu SY, et al (2012). Prevalence of human papillomavirus and cervical intraepithelial neoplasia in China: a pooled analysis of 17 population-based studies. Int J Cancer, 131, 2929-38.

Zhao R, Zhang WY, Wu MH, et al (2009). Human papillomavirus infection in beijing, people's republic of China: a populationbased study. Br J Cancer, 101, 1635-40. 